\title{
La Mise à nuit de la métaphore dans la poésie de René Char
}

\author{
Nathalie Riou \\ Université de Nantes
}

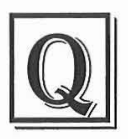

uand nous parlons, souvent nous oublions ce qui est - et la poésie, qui non seulement parle mais imagine et métaphorise, approfondirait cet oubli si elle ne lestait pas en même temps la parole de tout son poids sonore et rythmique : là-bas où on n'est jamais allé, ici comme on ne l'a jamais si intensément senti. Et il y a par ailleurs le lest, plus immatériel, d'un " cœur " traversant les niveaux de parole, biographique, fictif, métaphorique et/ou spéculatif. Le poème est donc tendu entre un surenchérissement de l'oubli du monde et une permanence du corps palpitant d'un sens, qui part peut-être du monde même.

La nuit est ce " peut-être " car elle est la part privilégiée du monde qui est plus qu'un thème, dit la langue obscure des poèmes nocturnes de René Char. La nuit dit toujours que quelque chose est nécessairement oublié dans la langue mais qu'en le disant et en le faisant sentir, une autre présence est possible. Le morceau poétique est, à sa manière, un météore : c'est cette " manière " qui sera l'objet de cette étude où, me penchant sur deux poèmes distants de plus de cinquante ans, j'essaierai de montrer que Char puise, dans une interrogation sur la nature du monde et du temps, son art poétique.

\section{En lisant "L'Historienne » : le corps de la métaphore}

L'Historienne

Celle qui coule l'or à travers la corne

Qui crève la semence

Mange aux pôles

Dort au feu de terre 
L'expression d'épouvante du visage du carrier

Précipité dans la chaux vive

Asphyxié sous les yeux d'une femme

- Son dos aux veines palpitantes

Ses lèvres de fleuve

Sa jouissance grandiose

Tout ce qui se détache convulsivement de l'unité du monde

De la masse débloquée par la simple poussée d'un enfant

Et fond sur nous à toute vitesse

Nous qui ne confondons pas les actes à vivre et les actes vécus

Qui ne savons pas désirer en priant

Obtenir en simulant

Qui voyons la nuit au défaut de l'épaule de la dormeuse

Le jour dans l'épanouissement du plaisir

Dans un ciel d'indifférence

L'oiseau rouge des métaux

Vole soucieux d'embellir l'existence

La mémoire de l'amour regagne silencieusement sa place

Parmi les poussières ${ }^{1}$.

\section{INSÉPARABILITÉ DU CORPS ET DE LA PENSÉE}

Le poème, rappelle Jean-Claude Mathieu, appartient à un cycle sadien de huit poèmes du Marteau sans maître: deux d'entre eux étaient " à l'origine dédiés à Maurice Heine, qui poursuivait au même moment sa publication des œuvres de $\mathrm{Sade}^{2}$ " et tout particulièrement Les Cent vingt journées de Sodome, dont le rouleau manuscrit de douze mètres venait d'être découvert en 1931. Dans ce roman, "l'historienne ", nommée la Duclos, joue les intermèdes, entre les actes sexuels, en racontant ses propres expériences de plaisir et de violence. Par ailleurs la fin du poème est aussi nourrie de la lecture des alchimistes : le retour à la rugueuse réalité - et à la syntaxe régulière - après l'amour, est signifié par un oiseau un peu philosophale, et les poussières gardent la pulvérulence des cendres vives. La deuxième strophe, quant à elle, se réfere à un accident qui eut lieu dans l'entreprise du

1 Le Marteau sans maître, p. 39. Toutes les citations de René Char se référeront à la réédition des Euvres complètes dans la collection de « La Bibliothèque de la Pléiade " (Paris, Gallimard, 1995).

2 La poésie de René char, I, Paris, Corti, 1984, p. 211. 
père de René Char, aux Plâtrières, un ouvrier étant réellement tombé dans la chaux vive. Le livre et la vie nourrissent donc le poème mais le lecteur, sans rien savoir de la Duclos ni de cet accident... peut très bien percevoir un éloge du sadisme, du plaisir de la souffrance éprouvée, ou infligée - car de "son dos " à " ses lèvres ", le sujet semble être tour à tour le carrier puis la femme, et surtout on va, mais de façon plus attendue, d'une scène vraisemblable vers une scène imaginaire : la métaphore monte pour ouvrir un récit d'ampleur épique : « lèvres de fleuve » et " jouissance grandiose ».

La scène est dramatisée et figée par les vers nominaux qui disent la catastrophe, accrue encore par les connotations : un ailleurs travaille souterrainement le texte. La métaphore in praesentia, "ses lèvres de fleuve », préparée par la " (ch)aux vive », " asphyxié " et " veine ", accroît l'ambiguïté entre homme et femme, entre masochisme et sadisme, et condense une comparaison à quatre termes, dont l'un est inconnu : les rives sont au fleuve ce que les lèvres sont au... L'image n'est pas " juste ", n'est pas claire, elle est localisable mais non définissable : c'est un climat épique qui est suggéré. Elle est facile en ce qu'elle met ensemble, sous nos yeux, deux réalités concrètes - il n'y a là aucune tentation métaphysique et elle semble même faite pour illustrer la thèse d'Aristote, selon qui l'art imite la nature, ou celle de Paul Ricœur qui voit dans le procès métaphorique même une élévation telle qu'elle se produit dans la mimèsis du récit, le muthos, au théâtre.

Dans La métaphore vive, paru en 1975, Paul Ricœur, interroge en effet et approfondit les différentes approches de cette figure centrale du lyrisme. Il part d'Aristote pour qui le mouvement métaphorique est premier, la comparaison venant après comme pour le ralentir. Dans la Rhétorique, Aristote évoque sa fonction argumentative dans l'espace de la parole, espace fragile que Ricœur commente ainsi : " Placée entre deux limites qui lui sont extérieures - la logique et la violence -, elle [la rhétorique] oscille entre deux pôles qui la constituent : la preuve et la persuasion ${ }^{3}$ ». La métaphore, proche du syllogisme par ressemblance ou vraisemblance, soit l'enthymème, est également tendue entre pensée et beauté : quelle bonne image donne à voir, encore, une pensée, déjà? Ricour se demandera même, à la suite de Gadamer, s'il n'y a pas un geste métaphorique à l'origine de notre pensée rationnelle qui distingue, et donc si le sens du "semblable » ne va pas de pair avec celui du " même » ou "différent ». La Poétique, quant à elle, montre que la métaphore relève d'une énonciation, lexis, qui intériorise, en quelque sorte, l'action de la tragédie. Et donc, selon Ricœur, le récit tragique, qui

3 La métaphore vive, Paris, Seuil, 1975, p. 46. 
imite, reconstruit et surélève le monde humain, ouvrirait le chemin à la métaphore dont l'intrigue serait le transfert. Dans la suite de l'étude, il oppose finement, à l'idée brute de substitution métaphorique, les nuances d'une tension entre l'actuel de l'énoncé et le virtuel antécédent, entre le "semblable " et la différence qui les distingue encore, entre le mot qui concentre la tension, jusqu'à l'oblitérer, et la phrase ou énoncé qui la touche et l'étend jusqu'au réel, entre l'être et le " n'être pas » du voir de l'image.

Dans les deux dernières parties, interrogeant le rapport entre métaphore, réel et pensée, il récusera Derrida qui pense que la pensée idéalisante s'approprie toujours le corps de la métaphore, celle-ci ne pouvant se retourner sur elle-même, car son corps ou signifiant s'est dissipé. Puis Ricour reviendra à Aristote, pour qui la métaphore met sous les yeux car " les mots peignent, quand ils signifient les choses en acte ${ }^{4}$ »cela contre Platon qui relie métaphoriquement le monde aux idées, le visible à l'invisible métaphysique. Heidegger, héritier critique de Platon, essaie également de penser " outre ", mais sans perdre de vue "la vue " du corps $e t$ de l'esprit : c'est cette métaphore qu'il prend pour exemple dans $L e$ principe de raison. Ricour le lui reprochera en ce qu' elle tendrait à confiner sa pensée dans le domaine philosophique - mais on peut aussi bien estimer qu'il est honnête de s'en tenir au monde qu'il connaît ou explore. C'est dans cet essai de 1962, que Heidegger lance : «le métaphorique n'existe qu'à l'intérieur des frontières de la métaphysiques ", car la métaphore engage ensemble, mais aussi en discorde, le corps et l'esprit. Ricœur, tout en critiquant la prétention d'Heidegger à envoyer promener toutes les métaphysiques antérieures, restera à l'écoute de sa pensée qui pense à l'intérieur de la frontière entre le corps de l'image et la pensée de l'image, pour qu'éclose la parole de l'Être... pas si loin des théologiens médiévaux qui, tel Thomas d'Aquin, cherchaient un énoncé métaphorique qui répondît à la nécessité d'approcher le divin tout en en respectant l'intégrité.

Cependant, pour Ricour, pensée et poésie peuvent se mesurer du regard et échanger en restant distincts : elles le doivent même car il y a une responsabilité à dire ses limites. Le spéculatif, à la recherche du " même " - tandis que le poétique dirait le " semblable " - serait aussi plus responsable de l'autre du langage, à savoir la réalité. Auparavant, Paul Ricour aura enrichi notre perception de la métaphore en donnant vie à la frontière qu'elle élargit. En effet, la métaphore tient entre la fusion et le discord des deux termes, puis à l'élévation ontologique « du sentiment à la

4 Cité par Ricœur in La métaphore vive, op. cit., p. 50; p. 388.

5 Cité par Ricour in La métaphore vive, op. cit., p. 126. 
fiction " qui est " la condition de son déploiement mimétique " et qui bat en brèche la distinction entre dehors et dedans, et enfin, elle poursuit, sans délire métaphysique, le sillon creusé par Aristote, entre l'être de l'action et l'être du possible : "Le poète serait-il alors celui qui aperçoit la puissance comme acte et l'acte comme puissance ${ }^{6}$ ?"

Si on revient maintenant aux "lèvres de fleuve ", on peut, avec Ricour, percevoir dans le transport de la métaphore comme un récit, mais, à son encontre, on peut souligner que le "comme " le distingue de la mimèsis du " muthos ". La métaphore s'apparente à un récit policier, à une équation avec inconnu, mais en differe essentiellement car il y a tension, comme il le dit bien par ailleurs, concomitance entre le métaphorisé et le métaphorisant, le " mort " et son " assassin ", l'aimé dépité et l'amant comblé, pour reprendre l'image de Nelson Goodman lorsqu'il dit que la métaphore est " une idylle entre un prédicat qui a un passé et un objet qui cède tout en protestant ${ }^{7}$ ». Mais surtout c'est la clé qui est à chercher, non ce qui est dans le coffre : à savoir la relation de ressemblance entre les lèvres et le fleuve. Derrière ce lien, il n'y a rien - sauf une autre manière de voir le réel, et non de l'oublier : par exemple, si nous entrons dans l'image, elle peut donner une plus grande présence à la fiction réaliste qui précède, du carrier tombé dans la chaux. Le lien métaphorique entre fiction réaliste et irréel, parce que nous le sentons, parce qu'aussi nous ne le saisissons pas complètement - le quatrième terme est flou - ouvre paradoxalement sur le réel. Enfin si le lien est premier, la distinction entre objet et pensée, entre visible et invisible, devient obsolète, non pas parce qu'elle n'est plus, mais parce qu'elle est « déjà-là » dans ce lien. Des lèvres au fleuve, il y a moins un rapprochement par ressemblance entre deux corps, que l'interrogation, moderne, de tout passage : il y a un impossible passage de l'un à l'autre, qui se réalise par un " même " interrogatif. Or si le passage d'un corps à corps interroge de la même manière que le passage du corps à la pensée, c'est parce que toute métaphore donne déjà à penser, qu'elle parle une "langue corporelle ", peut-être "déjà là ", dans le monde. Le rapport obscur entre deux éléments, même matériels, met ensemble le corps et la pensée dans une tension sentimentale.

\section{INSÉPARABILITÉ SENTIMENTALE}

Le premier quatrain du poème accroît l'ambiguïté car les quatre gestes qui caractérisent la relative indéfinie, " celle qui », amorcent un récit impossible.

6 Riccur, La métaphore vive, op. cit., p. 392.

7 Cité dans La métaphore vive, op. cit., p. 249. 
Aussi cherche-t-on à le "réduire " avec un sens qui lui serait antérieur : " celle qui » est une force qui va se dépensant sans cesse - "à travers » - qui " crève " ce que les autres couvent, qui vit entre le feu central, nourrisseur des volcans, et les extrêmes; elle est le désir qui dévore, le puma et la proie... D'emblée on tâtonne : l'antécédent est caractérisé par des gestes impossibles ou invraisemblables qui sont alors perçus comme métaphoriques. L'optique platonicienne pointe alors : le geste du corps symbolise, donne sur un sens. Cependant, l'instant reste essentiel, où le récit se transforme en besoin de sens, sens qui vient donc d'abord d'un désaccord avec le réel vraisemblable : l'aller du temps narratif est malmené et se retourne sur le lien passé avec un métaphorisé soupçonné. L’obscur nous suit comme une ombre " déjà là », peu à peu visible. Par ailleurs, le corps poétique travaille contre cette idéalisation : entre indétermination de l'antécédent et inachevé de la syntaxe, sans antécédent défini ni suite verbale, nous n'avons qu'un tronc de phrase, nous sommes inachevés. Ceci creuse l'hésitation entre récit insensé et récit symbolique, entre corps délirant et unité du sens. Il n'y a donc pas figement allégorique dans "L'Historienne », car le vers, trajectoire nominale, pose plus intensément la tension engagée par le poème, morceau de beau sens peut-être arraché au monde.

Plus énigmatique encore apparaît la seconde relative indéfinie :

Tout ce qui se détache convulsivement...

Elle semble inverser une parturition - c'est un enfant qui met au monde et elle semble faire se rencontrer le corps de l'homme et le corps cosmique d'un météore, qui « fond sur nous à toute vitesse ». La personnification est nette mais pas le lien avec un élément antérieur virtuel : nous ne savons pas quel sens ou quel objet est indiqué. À nouveau sans début ni fin, suggérant la chute d'une comète ou une naissance, l'image suggère le vers même. Nous voyons un peu ou un instant : chaque vers lance, suggère une image vers du sens... C'est peut-être le noir roi, qui, dit René Char, " escorte les poètes 8 ", et c'est aussi le "voir comme " de Wittgenstein, dont les Investigations philosophiques, dans les années quarante, mettent en questions le Tractatus logico-philosophicus. À partir ainsi d'une image ambiguë - un " lapin-canard »-il développe toutes les nuances du trouble qui se produit en nous quand la perception n'est pas encore arrêtée : il fait

8 Fureur et Mystère, p. 230.

9 Tractacus logico-philosophicus, suivi de Investigations philosophiques, trad. Pierre Klossowski, Paris, Gallimard : 1961; coll. « Tel» : 1993; p. 325-361. 
varier les différentes pistes qui s'amorcent dans ce ralenti entre le corps percevant et l'esprit sur le point de choisir... Ricœur, qui évoque ces pages, rappelle que la métaphore, in absentia du moins, differe cependant de l'image double, car elle sous-tend une autre figure qui est bien absente : le lapin et le canard sont dans le dessin tandis que la femme est et n'est pas derrière la rose métaphorique. Et bien sûr le "voir comme ", en métaphore, est imaginaire : c'est plutôt un "voir comme voir ", qui, quand il est obscur, rend d'autant plus perceptible le corps de l'imaginaire. Wittgenstein interroge tour à tour, le "voir comme " puis le " vouloir dire ", quand une pensée serait là en puissance mais non manifeste : l'instant où le lapin se distingue du canard est aussi infime et confondant que l'instant où le mot sur le bout de la langue va prendre corps, et que l'instant où le visible de la métaphore va prendre une autre visibilité... Que voyons-nous et que comprenons-nous quand nous lisons :

Tout ce qui se détache convulsivement de l'unité du monde

De la masse débloquée par la simple poussée d'un enfant

Et fond sur nous à toute vitesse?

Contrairement à " celle qui ", qui pouvait évoquer la femme du titre, le " tout " est vraiment indéfini, à gauche au regard platonicien, ou vraiment à droite au regard aristotélicien : à la fois d'un corps " convulsif " et d'une " unité " philosophique, parturition humaine et cosmique. Nulle lecture ne peut arrêter le virtuel de l'image : ce qui est en dessous, nous ne le savons pas, ne le comprenons pas, ne le voyons pas; nous expérimentons... On cherche quelque chose qu'il semble possible de connaître ou comprendre - car, après tout, on comprend chaque mot et chaque relation grammaticale - on cherche un "même autre ", comme on cherche à mettre un nom sur un visage connu et pas reconnu, ou - si on met l'accent sur l'autre - reconnu et pas connu. Cet interstice qui dure est le «sujet» de cette métaphore.

Ainsi lors de la rencontre du corps imaginaire et de la pensée, dans le métaphorisant qui tend un lien de ressemblance obscur avec quelque antécédent, un sentiment étincelle. Le sujet de la métaphore est donc bien sa trajectoire, ce qui vraiment nous fait hésiter entre là-bas et ici. Le « voir comme " de Wittgenstein, qui laisse indécise la pensée dans la perception, a un équivalent dans la métaphore puisque c'est ce que l'on ne voit pas

10 Tractacus logico-philosophicus, suivi de Investigations philosophiques, op. cit., p. 354. 
bien, en imaginaire, qui appelle à un équivalent, pas encore séparé de la pensée - conflit alors sentimental. La métaphore quion ne comprend pas est lyrique.

À propos de notre impossibilité à savoir ce que nous pensons nousmêmes, sur l'impossible rencontre du savoir et de la pensée, Wittgenstein glissera cette parenthèse : "Tout un nuage de philosophie condensé dans une goutte de grammaire ${ }^{10}$ ». Avec l'absence métaphorique, c'est tout un orage de philosophie qui gronde. Car c'est une chose mystérieuse que de voir un peu en imaginaire, de concevoir un peu, et en même temps de "sentir » qu'on ne voit pas quelque chose... La métaphore de Char n'est pas dans une pacifique mimèsis car le point commun est le plus souvent problématique; le métaphorisé rayonne derrière ce qui apparaît, non comme quelque sacré glorieux, mais vraiment comme les rayons d'un soleil morcelé... La métaphore de Char est violente parce que pensée et corps ne vont pas ensemble harmonieusement, et parce qu'en même temps, plus elle donne à sentir cette limite douloureuse, plus le poème est tendu entre imaginaire et réel. Au point aussi, que le temps semble parfois inversé : l'image crée le modèle...

Contrepoint du vide auquel je crois ${ }^{11}$.

Et si nous ne pouvons pas nous reposer dans la métaphore obscure, violente, moderne de René Char, n'est-ce pas aussi parce que, comme la nature, elle ne s'arrête pas? A la toute fin des Investigations philosophiques, Wittgenstein se demandera : « Si la formation de concepts peut s'expliquer à partir de faits de la nature, alors ce qui devrait nous intéresser, au lieu de la grammaire, ne serait-ce plutôt ce qui dans la nature constitue la base de la grammaire ${ }^{12}$ ? » C'est vers là que va notre conclusion.

\section{En lisant « L'Amante » : le cœur de la métaphore}

\section{LA NUIT MAINTIENT LES CONTRAIRES ENSEMBLE}

René Char dédicace son dernier poème à Marie-Claude Char, rencontrée en 1980; alors qu'ils se marient en 1987, Éloge d'une Soup̧̧onnée est sur le

11 "Vers l'arbre-frère aux jours comptés ", La Parole en archipel, p. 359.

12 Tractacus logico-philosophicus, suivi de Investigations philosophiques, op. cit. p. 362 . 
point de paraître : l'outre-tombe ouvre un outre destin, le testament brûle dans un brûlant épithalame.

\section{L'Amante}

Tant la passion m'avait saisi pour cette amante délectable, moi non exempt d'épanchement et d'oscillante lubricité, je devais, ne devais pas mourir en sourdine ou modifié, reconnu des seules paupières de mon amante. Les nuits de nouveauté sauvage avaient retrouvé l'ardente salive communicante, et parfumé son appartenance fiévreuse. Mille précautions altérées me conviaient à la plus voluptueuse chair qui soit. À nos mains un désir d'outre destin, quelle crainte à nos lèvres demain ${ }^{13}$ ?

La violence contre-nature est ici moins présente : Char va plutôt contre la société quand, avec un sourire de vieux faune, il dit simplement sa lubricité. Cependant, la sauvagerie retrouvée des nuits provoque le néologisme "d'outre destin » qui va contre l'absolu de la nature, et donc la mort. Quand, dans "L'Historienne ", la syntaxe inachevée semblait ramener le langage à une brutalité "naturelle ", il y a ici une violence plus distanciée, du côté de la logique. Grammaticalement, l'énoncé, « je devais, ne devais pas mourir en sourdine ou modifié, reconnu... ", est complexe : quatre relations jouent entre verbes et compléments (je devais mourir en sourdine, ou modifié, ne devais pas mourir en sourdine, ou modifié...). En même temps, la parataxe ainsi que l'ellipse du " je » entre " je devais, ne devais pas " laisse entendre un " et ", scandaleux au niveau de la logique, et donc une autre relation encore avec les compléments. On peut donc aussi lire : « je devais et ne devais pas mourir en sourdine, ou modifié " : le mourir/ne pas mourir est en relation intrinsèque grâce surtout à ce "modifié ", qui prend une valeur positive suggérée par la juxtaposition du heureux " reconnu des seules paupières... ". D'où une septième relation encore... Cette analyse décompose ce que nous entendons ensemble, comme les lignes d'un morceau de musique, et définit ce qui en train d'émerger comme une figure d'une peinture abstraite... mais elle peut aider à interpréter une relation entre l'obscur du monde et l'obscur de la poésie.

La conjonction d'une affirmation et d'une négation attaque l'essence de la logique. Elle remonte à Héraclite et à bien avant encore : à l'homme qui vit jour et nuit. Le jour affirme le monde en le montrant, la nuit le nie en l'obscurcissant - le « $\mathrm{n}$ " dans nos langues latines semblant même

13 Éloge d'une Soupçonnée, p. 849.

Riou 67 
rapprocher le non et la nuit. Mais pourquoi mettre ensemble ce qui se succède pacifiquement dans le temps? Le jour nous veillons, la nuit nous dormons. Mais parfois on rêve, on voit en imaginaire, c'est un " voir comme voir ». Il arrive aussi qu'on reste éveillé les yeux ouverts, et que, en se retournant sur son oreiller, on retourne le monde... L'affirmation du monde, par le rêveur, ou par l'insomniaque, persévère dans la nuit. En retour, dans le chassé-croisé du poème, qui va outre la nature, la négation de la nuit persévérera aux côtés de l'affirmation du jour, pour que, par exemple, se réfléchisse, dans l'atelier de Giacometti, un

beau visage sans antan qui allait tuer le sommeil, dans le miroir de notre $\operatorname{regard}^{14}$.

C'est une mise en abyme infinie car on sait aussi, depuis Freud, que la syntaxe du rêve peut coordonner deux oppositions, contrairement à la raison du jour qui nous aveugle d'une lumière absolue :

Il eût suffi d'un non lumineux pour indéfiniment allonger et élever nos doigts sur l'étendue et sur les choses. La pierre milliaire où se dépensait devant les joncs toute source à saisir est maintenant mutilée ${ }^{15}$.

\section{LES « CHASSÉS-DÉCROISÉS » DE LA NUIT}

On rencontre plus souvent dans la poésie de Char, une déconstruction des oppositions telle qu'on peut la lire dans " en sourdine ou modifié, reconnu... ". La coordination des compléments trompe notre attente deux fois : on attendait après " en sourdine ", un élément en contraste comme " avec éclat, intensité... ", qui aurait poursuivi le plan métaphorique et logique. Le gain de ce jeu de dissymétries est que nous comblons les cases nouvelles suggérées : " sourdine " signifie donc inchangé, et "modifié » indiquerait la voix audible du poète, suggérant alors que vivre jusqu'au bout, c'est-à-dire changer, se pourrait grâce au regard parlant de l'amour... On reconnaît le chiasme ou "chassédécroisé ", tel qu’on vient de le voir avec les "lèvres de fleuve ». Contre l'ascendance de Hugo, Char nuance les oppositions en construisant des rapports à quatre termes, elliptiques et rompus, en suggérant une tension

14 "Célébrer Giacometti », Le Nu perdu, p. 431.

15 " Mutilateurs ", Aromates chasseurs, p. 523. 
entre sonorité et sens. Il interroge les " chassés-décroisés naturels " de l'ombre et des étoiles - étoiles dont la clarté éclatée est une réplique asymétrique à l'ombre qui lie les êtres à la terre. La bougie de l'homme apparaît comme une plus juste répartie, si n'était son humanité - mais humanité qui, en retour et à nouveau, fonde la figure entre nature et artifice visible. Enfin, cette opposition minutieuse et surtout elliptique nous embarque, bon gré mal gré, dans la profondeur d'un cours détourné du temps.

La seconde surprise de " en sourdine ou modifié » est de conjoindre métaphore et terme de logique réaliste. L'hétérogène de l'association est fréquente dans notre langue où souvent, le corps s'effaçant, on ne l'entend plus mais ici le choc est maintenu car il est redoublé par le chassé-croisé de l'opposition. Le sujet du poème est à nouveau le conflit du réel et de l'imaginaire métaphorique, tel que peut le suggérer la lune qui s'oublie parfois dans le ciel le jour.

Par ailleurs en comparant récit et métaphore, on peut fonder davantage le lien de celle-ci avec la nuit. Le récit, en donnant le monde immédiatement, est plus que l'illusion de jour car il donne sens au monde; le récit est un jour dans le jour. Lié à la chronologie, il va sous le signe du soleil, qui fait le jour et le divise en heures. La poésie lyrique, quant à elle, refuse les parenthèses, en persévérant dans le corps de la langue et le sentiment personnel, qui font que le réel n'est jamais absent. Elle apparaît donc comme un "bâton » dans le récit qui tourne, d'autant plus que la métaphore obscure fait obstacle à l'imitation, à l'identification, et au souci du temps qui va, en cachant là-bas... En effet la métaphore tend au présent : quelque chose est ici en puissance sous nos yeux, couvant une relation complexe. Le poème de Char, quand il se resserre sur un aphorisme, indique bien le vou de faire du présent une plénitude autant qu'un passage. Le poème incline donc à la nuit en ce que la nuit n'a pas d'astre qui à la fois la fasse et la mesure - c'est même un essentiel et naturel " chassé-décroisé " : ce qu'est le soleil au jour, rien ne l'est à la nuit. Le "en puissance " de la métaphore est en consonance avec l'absence d'agent temporel de la nuit, propice pour dire l'instant, ou l'éternité.

\section{LA NUIT COMME ORIGINE COMMUNE DE DEUX MÉTAPHORES}

Je poserai qu'il y a deux grandes sortes de métaphores qui induisent deux sortes de lecture : celle qui tend un lien entre deux concrets, que l'on voit ou pas; celle qui tend un lien entre un concret et un spirituel, que l'on comprend ou non. La première qui met en fraternité les objets sous-tend 
une vision heureuse et naïve du monde, la seconde une vision platonicienne idéaliste qui oblitère le métaphorisant concret. La poésie moderne travaille à récuser ces dualités, et Char depuis la limite qui les sépare. La fin du poème "Les Trois Sours ", qu’il considérait un peu comme son art poétique, le répète :

\author{
Restez fleur et frontière \\ [...] \\ La plaie qui rampe au miroir \\ Est maîtresse des deux bouges. \\ [...] \\ Tout s'évanouit en passage ${ }^{16}$.
}

D'une part la métaphore concrète moderne est souvent surprenante et peu réductible à une analyse logique et si, comme on a vu, on ne peut réduire la métaphore, pourtant in praesentia, des "lèvres de fleuve", c'est peut-être qu'il y a déjà en elle du spirituel qui nous échappe : alors s'invente une autre manière de voir, on entrevoit un autre réel. D'autre part la métaphore spirituelle est souvent difficile à immobiliser dans un sens clair, en raison de la syntaxe heurtée, elliptique, de la polysémie... On hésite entre deux sens, entre quatre sens et, pour ne pas s'y perdre, on reste accroché au métaphorisant concret : seuls demeurent sûres les marques physiques. Le sens est encore concret : alors s'invente une autre manière de comprendre, on conçoit une autre pensée.

Si on prend la suite du poème de "L'Amante ", la métaphore, au prime abord, est facile :

Les nuits de nouveauté sauvage avaient retrouvé l'ardente salive communicante, et parfumé son appartenance fiévreuse.

L'éros heureux des deux amants s'étend à la nuit d'une nature personnifiée, épique. La métaphore est à nouveau irréductible à la raison en ce que la salive de la nuit n'a pas d'antécédent réaliste; par ailleurs, une amorce d'oxymore, entre le feu d " ardente " et l'eau de la "salive ", l'approfondit. Tout s'aime et s'emmêle : le rythme des consonnes et des voyelles (le " $\mathrm{v}$ ", le « an »...), les éléments du feu, de l'eau et de l'air, les sens avec le goût, la vue, l'odorat, le réel et l'imaginaire... La métaphore file ensuite une légère personnification : le parfum d'un mimosa la nuit aura peut-être baigné les

16 Fureur et Mystère, p. 251. 
amants, et l'alexandrin, où consonnent le " par » et le « $\mathrm{f}$ », va fondre le monde de l'amour dans une belle harmonie imitative... Mais ce n'est pas sûr : il est possible que la nuit, en parfumant, spiritualise un peu la possession de la femme, que le "parfumé " adoucisse l'" appartenance fiévreuse ». On perçoit et conçoit alors qu'un sens, le plus immatériel avec l'ouïe, indique à peine une possible transcendance de la nuit. On hésite entre une légère personnification de la nuit, réaliste, et une métaphore qui indique une transcendance. Le " corps à corps " métaphorique pense en puissance : une même expression, "parfumé son appartenance fiévreuse ", tend un lien entre corps réel et personnification, et en même temps entre corps imaginaire et transcendance. De "l'ardente salive " à " fiévreuse ", on est doucement passé du réel à un "outre réel ", d'un corps signifiant vers un sens du corps, vers une libre transcendance. On entrevoit le parfum concret entre la nuit et la femme, et on conçoit le parfum spirituel entre réel et irréel. La métaphore de Char n'est pas à l'intérieur des limites de la métaphysique car elle est la limite même entre physique et métaphysique : nous l'entrevoyons en la concevant et vice versa. Cette limite est notre demeure.

\section{ENTRE LE CORPS ET L'ESPRIT, BAT LE CCEUR DE L'Être ET DE LA NUIT}

Le "sujet " lyrique s'altère dans le corps de la parole poétique : lorsque l'amant parle de parfumer une " appartenance fiévreuse "... une tension naît entre signifié et signifiant, dans le cours rythmé d'un vers. Un je s'est risqué dans le corps commun de la langue qui donne communément le monde : est refondé un lien entre le concret du son - qui touche au monde - et l'abstrait du sens. Et retendant ainsi un lien dans le cours de l'énoncé, Char opère des chassés-croisés de sens de telle manière que la rencontre entre le corps et le sens devient plus intime et plus débattue : le sens devient le même du corps qui devient l'autre d'un corps proche... Cette tension du sens et du corps croise la tension engagée par la métaphore entre densité du présent et aller du récit, verticalité et horizontalité. Or, dans ces noces de la nuance et du contraire, parce qu'elles sont difficiles, le lien devient sentimental :

Quand nous disons le : le coeur (et le disons à regret), il s'agit du coeur attisant que recouvre la chair miraculeuse et commune, et qui peut à chaque instant cesser de battre et d'accorder ${ }^{17}$.

17 Les Matinaux, p. 332. 
Cette réflexion, qui « limite " le coeur au feu et à la finitude, appuie sentimentalement sur le corps : " chair miraculeuse et commune".

Puis, tout en remettant ainsi en jeu le corps et la pensée dans la langue poétique, la lyrique moderne interroge le sujet de la parole : un " qui vive ici? " habite la plupart des poèmes. Quand le théologien sépare le corps sexuel et le spirituel, quand le philosophe analyse que l'homme perçoit le monde en relation avec l'autre, un poète dit d'un seul trait :

Nous qui voyons la nuit au défaut de l'épaule de la dormeuse Le jour dans l'épanouissement du plaisir ${ }^{18}$.

Un seul être nous manque et la nuit est ici; que le plaisir nous comble, et voici le jour qui s'épanouit - ou aussi bien l'inverse, car ce qui importe est le rapport. L'abord un peu égocentrique, un peu hugolien, de ces vers, s'efface dans l'élargissement du nous, dans la critique du religieux, et dans la mise en avant du corps sur le coeur, de "voyons " à " plaisir ". Et en disant que l'amour donne le pouvoir de voir et de ne pas voir le monde, on rencontre en même temps l'autre et le monde.

Mais si on pose que la relation est première, elle peut s'inverser. L'être, le "corps-cœur", et le monde : cette relation triangulaire devrait pouvoir s'ouvrir depuis le cœur du monde même. Ainsi, de l'autre côté de la tension - et de l'œuvre - il y aura : " les nuits de nouveauté sauvage avaient retrouvé l'ardente salive communicante... », la nuit étrange se faisant sujet de l'énonciation. Et si «L'Amante » nous touche, malgré l'insistance des hyperboles - «tant », « seules », « mille »... - n'est-ce pas justement parce qu'elles sont liées à cette étrangeté de l'énonciation? Char appuie sur le cœur mais on entrevoit et conçoit que le coeur viendrait d'ailleurs, comme tout à l'heure le parfum apparaissait un instant émanant d'une transcendance... On peut alors comprendre pourquoi il ne s'est pas dit reconnu des yeux de son amante mais de ses "seules paupières ": de " peau " et de " pierre ", elles sont peut-être déjà engravées de poésie, mais elles sont d'abord à la limite du monde et d'une perception; elles font ou ne font pas l'amour, mais elles font toujours le jour et la nuit du monde... La synecdoque, qui métaphorise l'indécision entre la femme en chair et en os et la relation poétique, altère un peu plus nos habitudes de pensées déjà mises à mal dans cette phrase complexe dont le schéma logique, avec des branches asymétriques, miné par la coexistence d'une affirmation et d'une négation, est un peu plus inabouti. Et c'est pourquoi, par la suite, les

18 "L'Historienne ", Le Marteau sans mâ̂tre, p. 40. 
"précautions " sont sujet grammatical et étrangement " altérées " : soit par le désir, soit par la nuit poétique retrouvée. On ne peut démêler les mots d'amour et ceux du poème. La première pensée du premier homme n'est pas plus incroyable que le premier de ces « je t'aime " poétiques, à la fois privés et pourtant colportés et pourtant rapportés à mille et un inconnus, et peutêtre légitimement car on ne sait quelle voix démêler dans « ... quelle crainte à nos lèvres demain? " : voix du poète, du poème, ou nôtre?

Cette " main dans la main » qui paraphe poétiquement tout l'œuvre de Char est le trait commun qui unit deux êtres au monde, mais aussi l'être et le monde, c'est-à-dire de quoi aller en métaphore. Dans la perception lyrique, entière, du monde, rien n'est seul, si bien qu'on perçoit le monde avec le cour et la pensée, qu'on pense avec le corps ému... mais prééminent est le cœur, dont l'heureuse orthographe française dit bien « le couple enlacé du $\operatorname{mot}^{19}$ ", l'eidétique du lien. Il y a des "isoloirs ", dans nos sociétés, où l'on peut distinguer la pensée - ce sont nos livres de philosophie - d'autres où l'on peut distinguer le corps - ce sont nos stades - mais où voit-on qu'on puisse isoler le cœur de son expression physique et de sa pensée?

De la même manière qu'il n'y a pas d'astre qui fasse la nuit, il n'y a pas de sujet qui fasse la parole poétique - sinon sous forme de question, question inquiète et nécessairement sentimentale... C'est pourquoi aussi la progression va de soi, qui fait que le poète est d'abord assez " riche de nuit " pour persévérer contre les limites de la mort, puis qu’à l'ouverture du dernier recueil, la nuit mortelle ayant mordu sur la vie, il lance doucement :

A quoi bon s'éclairer, riche de larmes ${ }^{20}$ ?

Comme si les larmes coulaient de ces paupières entre nuit et amour... Ainsi le propre d'une poésie lyrique moderne serait de dire le " cœur " non comme siège individuel des émotions, mais comme lien nécessaire, comme battement commun entre l'être et le monde. Le poète dit tout, non à partir du corps ou de la pensée mais à partir de leur rencontre où bat le premier cœur. Et peut-être peut-on concevoir un peu la dernière méditation de Char qui s'interroge sur comment dire jusqu'au bout la poésie avec spontanéité, avec et malgré toute notre intelligence :

19 "Maintien de la reine ", Placard pour un chemin des écoliers, p. 99.

20 "Sa main froide ", La Nuit talismanique qui brillait dans son cercle, p. 504; « Riche de larmes », Éloge d'une Soupçonnée, p. 839. 
Dans ce monde transposé, il nous resterait à faire le court éloge d'une Soup̧onnée, la seule qui garde force de mots jusqu'au bord des larmes ${ }^{21}$.

Et la seule qui garde lumière - ou faiblesse - de larmes jusqu'au dernier poème.

\section{LA MÉTAPHORE OU l'ÉCLAIR AU NU DE LA NUIT}

On peut maintenant comprendre que la métaphore, unité d'action dans le monde transposé de la poésie, puisse apparaître comme le transport du coeur, de l'homme au monde, puis du monde à l'homme... Elle est la figure où l'inversion doit se produire. La métaphore qui transforme la relation prosaïque entre les mots, les choses, et le sens, en relation tendue d'amour cherche à capter la trace de ce qui n'a pas encore eu lieu, la parole de ce qui nous dépasse. Alors qu'il nous semble que le métaphorisé précède, qu'il est peut-être même prémédité par le poète arachnéen ${ }^{22} \ldots$ soudain nous l'entrevoyons et le concevons mais tel que nous ne l'avons jamais vu... De même l'éclair...

L'éclair fendant la nuit, emblème de la poésie de Char, apparaît comme le ravissement du visible, plus vrai d'être un instant, d'être mortel. La métaphore serait cet éclair quand, de la rencontre de deux éléments, comme deux nuages, dans le cours nocturne du vers où se débattent corps, sens, sujets..., elle nous donne à entrevoir et concevoir le monde autrement... Le métaphorisant, imaginaire, est à la fois contenu horizontalement par le débat du vers poétique, et tenu dans la tension verticale vers quelque chose qui serait... : rencontre si intense qu'un instant le métaphorisé apparaît, plus vrai qu'il n'a été, et donc comme neuf, comme venant après le métaphorisant :

Les dieux sont dans la métaphore. Happée par le brusque écart, la poésie s'augmente d'un au-delà sans tutelle ${ }^{23}$.

Bien sûr, l'éclair montre le même monde que le soleil, il introduit du différent dans le même et donc il ne " métaphorise " que parce qu'un

21 "Bestiaire dans mon trèfle ", Éloge d'une Soupçonnée, p. 843.

22 " Au seuil de la pesanteur, le poète comme l'araignée construit sa route dans le ciel. En partie caché à lui-même, il apparaît aux autres, dans les rayons de sa ruse inouïe, mortellement visible. » « Partage formel », Fureur et Mystère, p. 165.

23 A Faulx contente, p. 783. 
instant l'éphémère tonne si fort qu'il semble altérer le monde, être la parole émue du temps. Et pourtant l'opération de la métaphore, en indiquant un " même » problématique entre deux domaines, aboutit au même instant renversant quand le monde ( $\mathrm{du}$ domaine métaphorisé) nous apparait différent, sinon neuf : cet instant dégauchirait une transcendance, pour reprendre les mots de Char.

Aussi, l'éclair consonne avec la rhétorique de Char : ce qu'il a de plus abstrait, en relation avec l'homme, à savoir le temps de notre finitude, se retrouve dans les effets d'une métaphore. L'art de Char prend source et reçoit aval du plus abstrait de la nature, le temps. Tandis que Mandelstam se lave une nuit et voit une étoile disparaître dans l'eau de la cuve, il perçoit la terre comme plus vraie et plus épouvantable; de même elle apparaît plus vraie sous l'éclair de René Char mais à l'inverse - à nous qui vivons loin des totalitarismes - plus adorable. Ainsi ce qui n'est pas, le métaphorisant, entre pouvoir et impossible, renouvelle le monde, le métaphorisé, en suggérant un cœur :

Jadis l'herbe avait établi que la nuit vaut moins que son pouvoir ${ }^{24}$.

Comme si tout ce qui nous disperse trouvait un sens avec un monde qui parlerait enfin en premier, après avoir disparu en imaginaire. Et ainsi la métaphore n'imite pas le monde, ne fait pas les choses s'aimer naïvement : choses et sens débattent et ne s'entendent que dans le cœur soupçonné du monde.

Poursuivant la belle question de Novalis qui se demandait à qui ne plairait une philosophie dont le premier geste est un baiser, on pourra conclure, les lèvres s'ouvrant maintenant entre réel et imaginaire où s'entremêlent le corps et l'esprit, en se demandant : à qui ne plairait une poésie qui finit et ne finit pas dans un transcendant et très sonore baiser?

Commence à croire que la nuit t'attend toujours ${ }^{25}$.

C'est une note inaugurale, des environs de 1935, parmi les plus brèves de Moulin premier, et qui questionne le chemin même : on ne va pas du jour à la nuit, ni de la nuit au jour, mais plutôt on répond à une présence qui met à mal la notion de sujet même. Le soleil devenant feu de l'homme

24 " Jacquemard et Julia », Fureur et Mystère, p. 258.

25 Le Marteau sans maître, p. 72. 
métaphorise par excellence la métaphore qui met à mort le monde réel; à l'inverse la nuit est le « déjà-là » de l'autre du réel, il n'y a pas de progrès, de chemin, pas de recette pour la pénétrer ou plutôt en être pénétré. Si la poésie a un sens dans notre vie, elle est aux antipodes de l'ornement rhétorique : elle est, et si elle éclaire notre monde, elle est dans un déjà-là entre ici et ailleurs. Il est possible d'entendre et de voir une métaphore dont le métaphorisant donne et ne donne pas le métaphorisé, suggérant ainsi que l'imaginaire ouvre le réel : le métaphorisant fait le métaphorisé. De même que la nuit authentifie physiquement qu'il y a de l'obscur, elle est la vérité nue que le poème ne prémédite pas. Elle n'est pas l'inverse du jour mais autre : elle n'a pas de devenir chronologique, elle demande plutôt passivité et écoute, elle est maîtresse mais ne maîtrise pas. Elle règne dans la plupart des passages obscurs de la poésie de Char.

Je terminerai en laissant la parole à René Char, tout en souhaitant ne pas la lui avoir tout à fait prise; il s'agit de la fin du premier poème qu'il a publié à vingt ans :

Enfin de son pourpoint bleuâtre

Il fait signe à la nuit d'entrer ${ }^{26}$.

26 "Aumône d'hier ", in La Cigale uzégeoise, $n^{\circ} 3$, mai 1927. Le poème sera repris dans Les Cloches sur le cour (cité dans Char. Dans l'Atelier du poète, Paris, Gallimard, coll. "Quarto ", édition établie par M.-C. Char, 1996, p. 50). 